\title{
The effects of host genetic architecture on the gut microbiome composition of Chinook salmon (Oncorhynchus tshawytscha)
}

\author{
Mubarak Ziab ${ }^{1}$, Subba Rao Chaganti ${ }^{2}$, and Daniel Heath ${ }^{1}$ \\ ${ }^{1}$ University of Windsor Faculty of Science \\ ${ }^{2}$ University of Michigan
}

April 27, 2020

\begin{abstract}
The microbiome community consists of microbes living in or on an organism and has been implicated in both host health and function. Environmental and host-related drivers of the microbiome have been studied in many fish species, but the role of the host genetic architecture across populations and among-families within a population is not well characterized. Here, Chinook salmon (Oncorhynchus tshawytscha) were used to determine inter-population differences and additive genetic variation within populations for gut microbiome diversity and composition. Specifically, hybrid stocks of Chinook salmon were created by crossing males from eight populations with eggs from an inbred line of self-fertilized hermaphrodite salmon. Based on high-throughput sequencing of the 16S rRNA gene, significant gut microbiome community diversity and composition differences were found among the hybrid stocks. These differences likely reflect divergent selection shaping the gut microbiome and its co-evolution with the host. Furthermore, additive genetic variance components varied among hybrid stocks, indicative of population-specific heritability patterns, suggesting the potential to select for specific gut microbiome composition for aquaculture purposes. Determining the role of host genetics in shaping their gut microbiome has important implications for predicting population responses to environmental changes and will thus impact conservation efforts for declining populations of Chinook salmon.
\end{abstract}

Keywords: microbiome, genetic architecture, host genetic variation, selection, additive genetics, additive population variance

\section{Introduction}

Despite the wide recognition of the gut microbiome as a critical factor in host fitness, there is still a lack of studies partitioning the relative contribution of the host genetics architecture and environmental factors involved in shaping its composition and function. In addressing the factors that shape the host's gut microbiome (hereafter "microbiome") composition, it is important to consider the broad effects of the environment, evolutionary forces reflective of host genetic divergence, and host genetics-by-environment interactions. With over 32,000 described species (Eschmeyer \& Fong, 2015), fish comprise more than half of the known vertebrate species and encompass a wide range of phenotypes, life histories and ecologies (Neslon, Grande \& Wilson, 2016). Thus, even with a wealth of published fish microbiome studies, the effects of host genetics, the environment, and their interactions on the microbiome composition remain poorly understood (Wong \& Rawls, 2012; Bolnick et al. , 2014; Ghanbari, Kneifel \& Domig, 2015). Host genome variation is expected to play a role in shaping the microbiome, as it is responsible for encoding intestinal mucosa and immune factors that play essential roles in the establishment and maintenance of the microbiome (Roeselers et al., 2011; Spor, Koren \& Ley, 2011; Romero, Ring \& \& Merrifield, 2014; Ghanbari et al., 2015). Using high throughput sequencing technology, the effects of host genetics on the microbiome have been investigated at various levels, ranging from species-level effects (Roeselers et al.,2011; Ye, Amberg, Chapman, Gaikowski \& Liu, 2014; Larsen, Mohammed \& Arias, 2014; Li et al., 2015) to among-population (Roeselerset al., 2011; Webster et al., 2018) and within-population effects (Webster et al., 2018). For example, at the species-level, individuals from 
three species of carp (Ctenopharyngodon idella, Carassius carassius andHypophthalmichthys nobilis ) reared in a common environment showed strong microbiome compositional differences, despite their taxonomic relatedness and common environment (Li et. al 2014). Gut microbiome composition and diversity differences have also been found at the population level, and have been attributed to genetic drift and bottleneck effects using zebrafish (Danio rerio ) (Roeselerset al., 2011) or to environmental variation and genetic divergence using zebrafish (Roeselers et al., 2011) and Atlantic salmon (Salmo salar ) (Webster et al., 2018). Finally, within-population effects were shown to explain less variation than among-population effects for microbiome composition in Atlantic salmon (Webster et al., 2018) but explained less variation than diet in shaping the microbiome of various rainbow trout families (Oncorhynchus mykiss ) (Navarrete et al., 2012). Differences among families within a population may be indicative of heritable components in the microbiome, defined as the proportion of phenotypic variance in a population attributable to additive genetic variance (Visscher, Hill \& Wray, 2008). Thus, host-based drivers of the gut microbiome often have a strong underlying genetic architecture, which may include among-species, among-population (within a species) or among-family (within a population) variance components; however, population effects are perhaps the least understood (Ghanbari et al., 2015). More importantly, there are currently no studies on inter-population effects on microbiome composition in fish using controlled environmental conditions. Collectively, the literature shows that the host genome plays a pivotal role in determining the composition of the microbiome across various fish species, but there is still a gap in our understanding of the variance components of host genetic effects on the microbiome in fish.

In addition to host genetics, it is known that the host environment affects gut microbiome establishment during various ontogenetic stages of fish development (Llewellyn et al., 2016; Bledsoe, Peterson, Swanson \& Small, 2016). Fish ingest water and particulate matter directly from their aquatic environment, which unquestionably affects their gut microbiome (Llewellyn, Boutin, Hoseinifar \& Derome, 2014; Ghanbari et al., 2015). Various environmental conditions such as diet (Naverrete et al., 2012; Wong et al., 2013; Websteret al., 2018), or fish rearing environments (Roeselers et al., 2011; Wong et al., 2013; Webster et. al 2018; Parshukovet al., 2019) also contribute strongly to microbiome variation. Thus, it is critical to consider the potential environmental factors and control for them while investigating non-environmental drivers of the microbiome (Goodrich et al., 2014a; Ghanbari et al.,2015).

Selection pressures are known to act on the host-microbiome interactions, leading to co-evolved microbiomes across host species over evolutionary timescales (O'Brien, Webster, Miller \& Bourne, 2019). These co-evolutionary dynamics may be demonstrated, for example, as genetic co-divergence of the host and their associated microbiome, as evident between ecotypes of Trinidadian guppies (Poecilia reticulata) (Sullam et al., 2015) and among Atlantic salmon populations (Webster et al., 2018). In addition, these patterns may be reinforced by the presence of strong metabolic complementarity between the microbiome and its host (O'Brien et al., 2019), as reported in diet and microbiome correlations across mammals (Leyet al., 2008), and in fish (Sullam et al., 2015). Therefore, the microbiome itself reflects evolutionary selection pressures acting at the host level and the microbial cell level (Leyet al., 2006). Despite this, evidence for reciprocal adaptation in host-microbiome systems as a result of bi-directional selection is weak, and more empirical work is needed to better understand co-evolutionary dynamics (Foster, Schulter, Coyte \& Rakoff-Nahoum, 2017; Koskella, Hall \& Metcalf, 2017). While host-microbiome co-evolution in fish has not been explicitly characterised, salmonids are known to show strong patterns of population divergence, consistent with local adaptation (Garcia de Leaniz et al., 2017). Putative locally adapted traits are exhibited across many populations of Atlantic and Pacific salmon, ranging from trophic ecology and feeding behavior to immune and metabolic function (reviewed in Fraser, Weir, Bernatchez, Hansen \& Taylor, 2011). Since the host itself exerts selection pressures on its microbial community through host related factors (Ley et al 2006), it is intuitive that if host populations were locally adapted to their environments, that microbiome differences would be observed among-populations as a consequence of variation in host-related factors. However, the role of host genetic architecture in determining fish microbiome composition is under-studied, especially at the among-population and among family (within-population) levels. Demonstrating among-population microbiome variation in a controlled environmental setting may therefore indicate the microbiome's co-divergence 
with the host genome.

Perhaps among the best studied genetic architectures of non-model animals are those of salmonids', including the Pacific salmon (Waples, Naish \& Primmer, 2019). In this study, we focus on the economically and ecologically important Chinook salmon (Oncorhychus tshawytscha), which are native to the North Pacific Ocean and grow to be the largest of the Pacific salmon species (Quinn, 2018; Ohlberger, Ward, Schindler \& Lewis, 2018). Chinook salmon are anadromous and semelparous, and many populations remain in coastal waters until they return their natal streams to spawn (Rounsefell, 1958; Quinn, 2018). Underpinning the ecological significance of salmon is their importance in nutrient cycling, freshwater and saltwater trophic ecology, community behavioural interactions, and evolutionary relationships (Hilderbrand, Farley, Schwartz \& Robbins, 2004). Salmon production contributes significantly to the Canadian economy: Over 123,000 tonnes of salmon were farmed in 2018 alone, accounting for over 1.1 billion Canadian dollars (DFO, 2018). While there are limited recent statistics to partition the economic contribution of Chinook salmon from that of all salmon species, they are the largest cultured species of salmon in BC and have domesticated in the province since the 1970s, accounting for a fifth of all farmed salmon stocks in the province (Kim, Withler, Ritland \& Cheng 2004). The mid- and distal- gut microbiome of farmed Chinook salmon from New Zealand was recently sequenced and Vibrionaceae was dominant in both (Ciric et al., 2018; Ciric et al., 2019). Furthermore, the diversity and composition of the gut microbiome in Chinook salmon is known not react to a soybean (plant) based diet (Booman et al., 2018). However, other than those studies, Chinook salmon gut microbiome dynamics research has not been reported.

This study aims to address two main questions: 1) do evolutionary forces reflective of genetic divergence among natural populations affect the microbiome composition in controlled hybrid crosses of Chinook salmon (Oncorhynchus tshawytscha)?, and 2) are there within-population sire effects that act on the microbiome differentially among populations, reflective of additive genetic variance (heritability) effects? Salmonids are known to lend themselves to traditional breeding designs, permitting us to partition genetic and environmental sources of variance (Lynch \& Wash, 1998). Here, we reared half-sib families from a single fully-domesticated and seven wild-domestic hybrid crosses of Chinook salmon in replicated pens to test for population and within-population additive genetic effects on gut microbiome diversity and composition. Measuring the extent of gut microbiome variation among and within populations is important in efforts pertaining to the management and conservation of salmonids (Garcia de Leaniz et al., 2007).

\section{Materials and Methods}

Field collections, breeding design and rearing environment

All fieldwork was carried out at Yellow Island Aquaculture Ltd. (YIAL), a Chinook salmon hatchery and organic-based farm located east of Campbell River on Quadra Island, Vancouver Island, British Columbia, Canada (Figure 1). Wild sourced eggs from Robertson Creek and milt from Big Qualicum were used to produce the seven-generation, fully-domesticated, stock of Yellow Island (YIAL), which has been in production since 1985. Eggs from 17 highly inbred female (offspring from a self-crossed hermaphrodite) were mixed, and subsets of the mixed eggs were individually fertilized using 10 sires from each of the domestic wild stocks of Chinook salmon (details of breeding in Semeniuk et al., 2019). This produced 80 full- and half-sib families belonging to seven outcrossed hybrid stocks (YIAL $\mathrm{x}$ Wild) and a fully inbred domesticated stock (YIAL $\mathrm{x}$ YIAL) (Figure 1). Consequently, the maternal line is identical for all crosses, but the paternal line for those crosses varies depending on the geographical origin of the paternal line. The aim of this breeding design was to minimize maternal effects (Heath, Fox \& Heath, 1999; Semeniuk et al., 2019), while allowing the characterization of the genetic architecture underlying the microbiome variation due to sire (additive genetic variation) effects, pen (environmental) effects, and hybrid cross or 'population' effects. Husbandry conditions are detailed in Semeniuk et al., 2019.

Sample collection, DNA extraction and next generation sequencing

A subset of 2 year-old fish ranging from 10 to 25 (mean size $=182 \mathrm{~g}$ ) was randomly selected from each pen (Supplementary Table 1), and the fish were humanely euthanized and sacrificed to sample the gut contents. 
To obtain gut content samples for DNA extraction, the body cavity was cut open with a sterile scalpel and the hindgut of each offspring was collected. The gut samples were immediately stored in RNAlater ${ }^{\mathrm{TM}}$ for transport to the research facility, where it was stored in the freezer at $-20^{\circ} \mathrm{C}$ until DNA extraction.

We extracted DNA from gut content of the distal intestine using commercially available E.Z.N.A Stool DNA Kit (OMEGA Bio-tek) following the manufacturer's protocol. Next generation sequencing library construction was completed in two steps as previously described (Heet al., 2017). Briefly, the universal primer set of 787F (V5F) (ATTAGATACCCNGGTAG) and 1046R (V6R) (CGACAGCCATGCANCACCT) was first used to PCR amplify the 16S rRNA encoding gene sequences containing the V5-V6 hypervariable regions. A short, Ion Torrent adaptor sequence was added to the 5' end of the forward (acctgcctgccg) and reverse (acgccaccgagc) primers. The PCR product was visualized for amplification success on a $2 \%$ agarose gel, and PCR product purification was then carried out using Agencourt AMPure XP beads (Beckman Coulter Genomics GmbH, Mississauga, ON, Canada). A second short-cycle of PCR was conducted to ligate adaptor and the barcode sequences to the amplicon using purified PCR product from the first round PCR. The second round of PCR used: forward primer UniA (CCATCTCATCCCTGCGTGTCTCCGACTCAG $X X X X X X X X X X$ GATacctgcctgccg), and reverse primer UniB (CCTCTCTATGGGCAGTCGGTGATacgccaccgagc), where the underlined sequence in UniA consisted of unique 10-12 bp barcode sequences necessary for the sample demultiplexing in sequence analysis and the lower-case sequence were the reverse compliment of the added sequence in the first primer set. Barcoded samples were combined based on PCR band intensity and a commercially available kit (GenCatch ${ }^{\mathrm{TM}}$, Epoch Life Science, Inc., Sugar Land, TX., USA) was used to purify the PCR product from incomplete amplicons and primer dimers. The final library was sequenced with an Ion Torrent Personalized Genome Machine (Thermo Fisher Scientific, Inc., Mississauga, Canada).

Sequence processing and data analysis

Sequence quality checks were initially conducted using personal genome machine (PGM) software (Torrent Suite v5.6) using default parameters to conduct the following tasks: 1) removal of mixed clonal libraries on Ion Sphere Particles (ISPs) known as polyclonals, 2) removal of low-quality sequences, and 3) removal of sequences with low quality data at the 3 ' end of the read.

Unless otherwise stated, all sequence processing was performed using the Quantitative Insights into Microbial Ecology (QIIME) pipeline, v1.9.1 with default parameters (Caporaso et al., 2010). Briefly, raw sequences were processed with a Phred quality score cut-off of 25 and then demultiplexed. Any raw sequence with one or more mismatches in the primer sequence were detected and excluded. In addition, forward and reverse primer sequences, and barcode and adapter sequences were removed. Chimeric sequences were detected using USEARCH v6.1 (Edgar, 2010) and excluded from analysis. To perform operational taxonomic unit (OTU) clustering, the open reference approach in QIIME was used with default parameters and a $97 \%$ sequence identity threshold. In this approach, clustering is completed with the UCLUST algorithm (Edgar, 2010), wherein a reference database was used to determine a cluster of sequences, and unassigned sequences were allowed to cluster de novo (Caporaso et al., 2010). To assign taxonomy to OTUs, alignment of candidate OTU sequences was completed in PyNAST (Caporasoet al., 2009) against the GreenGenes database (v13.8) at $90 \%$ sequence identity using UCLUST (Edgar, 2010).

After sequence processing in QIIME, 6,411,635 high quality sequences (out of 6,602,610 usable sequences) remained for analyses. After filtering unassigned taxa OTUs, and OTUs from Archaea, mitochondria and Chloroplasts, a total of 8,038 unique OTUs were identified, with 6,317,692 working sequences (out of $6,411,635)$ and used for all subsequent statistical analyses. After sequence filtering and alignment, any samples with 3,000 reads or less were dropped from all statistical analyses. The mean sample depth across all samples was 22,871 , and the range was 3,061 to 175,783 reads per gut sample. In the final analysis, a total of 278 gut samples were used (Supplementary Table 1).

Alpha Diversity analyses

To estimate diversity and richness of the microbiomes, the Shannon and Chao1 indices were computed for all samples in QIIME by rarefaction with 999 iterations using a 3000-sequence cutoff, and the average was 
calculated across all bootstrap runs. The purpose of this analysis was to ensure comparable estimates of alpha diversity across samples with non-uniform sequencing depth. Linear mixed effects models were used to test for the significance of cross, sire (nested within stock) and pen (nested within stock) effects on Shannon diversity and Chao1 indices. Multiple t-tests were conducted post-hoc in emmeanspackage (v1.3.5) (Lenth, Singmann, Love, Buerkner \& Herve, 2018) in R (v3.6.0) (R Core Team, 2016) to test for differences between pairs of crosses, and corrections for multiple tests were made using false discovery rate (BH; Benjamini \& Hochberg, 1995).

To explore differences in the microbiome community structure, OTUs with 3 reads or less across all samples were discarded from the analysis. Then, the number of sequences across all samples was normalized to relative frequencies using the cumulative sum scaling (CSS) technique (Paulson, Stine, Bravo \& Pop, 2013), and Bray-Curtis dissimilarity matrices were generated using adonis in the R (v3.6.0) (R Core Team, 2016) package vegan (v2.5-5) for beta diversity analysis (Oksanen et al., 2013). The CSS normalization method was chosen as it considerably enhances PCoA clustering (Paulsonet al., 2013) and outperforms other normalization techniques in clustering accuracy, especially for libraries with high variation in the pair-wise distance measures, such as observed in this study (Bray-Curtis) (Weiss et al., 2017).

To visualize patterns of differences in the microbiome composition among population crosses, principal coordinate analyses (PCoA) were performed on the Bray-Curtis dissimilarity matrix in PAST (v3.25; Hammer, Harper \& Ryan 2001). To simplify the visualization of a large number of samples $(\mathrm{n}=278)$ on the PCoA plots, the averages and $95 \%$ confidence intervals of the first two principle coordinates were calculated across crosses and used to construct a representative coordinate of each cross on the plots.

To characterize the microbiome composition among crosses, a list of all OTUs present in each stock was created and an intersection plot was created using the UpSetR package (v1.4.0) (Lex, Gehenborh, Strobelt, Vuillemot \& Pfister, 2014) in R (v3.6.0) (R Core Team, 2016). The intersection plot shows the OTUs found exclusively in certain stocks, and OTUs found commonly among multiple stocks.

To test for cross, sire and pen effects on the microbiome community structure, a nested form of the permutational multivariate analysis of variance (PERMANOVA) (Anderson, 2001) was used in the veganpackage (v2.5-5) using the adonis function on the Bray-Curtis dissimilarity matrices with 9999 permutations. The model was used to test for among-cross effects, among sires (within cross) effects, and between pens (within cross) effects. The mean sum square values were subsequently used to partition the variance explained due to all factors. Separate models were also constructed for each stock to partition the variance explained due to sire and pen effects within each stock separately, and to test for their effects. To further explore specific patterns in community composition differences in the microbiome among populations, ad-hoc test comparisons were conducted using 9999 permutations to quantitatively assess the differences among stocks using adonis in the $\mathrm{R}$ (v3.6.0) (R Core Team, 2016) veganpackage (v2.5-5). Corrections for multiple simultaneous ad hoctests were adjusted using BH (Benjamini \& Hochberg 1995).

OTU and bacterial family-level abundance analysis

To explore variation in the microbiome at the level of taxonomic groups, unique OTUs that were most abundant, and bacterial families (consisting of OTUs that were identified and collapsed to the family level), were used for differential abundance analyses. Unique OTUs and taxonomic families that were most abundant with 3000 and 1000 sequences or more, respectively, were selected for analysis. In total, 110 OTUs and 45 taxonomic families were used, accounting for $92.3 \%$ and $99.5 \%$ of all sequences and all sequences with assigned taxonomy, respectively. Cumulative sum scaling was used to normalize OTU and family-level data to account for differences in read-depths and allow for a meaningful differential abundance analysis (Paulson et al., 2013). The OTUs and taxonomic families of interest were fitted to zero-inflated linear mixed effects models (LMM), with population cross as a fixed factor, and sire and pen as random factors nested within cross using theglmm TMB package (v.0.2.3; Magnusson et al. 2017) in R (v3.6.0) (Brooks et al., 2017). The Akaike information criterion (AIC; Akaike, 1973) was calculated for models with competing zero-inflation structures (absent, constant, or population cross-specific; Brooks et al. 2017) and used to select the best 
model in the analysis (as suggested in Xu, Paterson, Turpin \& Xu, 2015). To test the statistical significance of fixed and random terms in the model, a reduced model for each term was used, and the change in the log likelihood between the models was compared against a $\chi^{2}$ distribution using the likelihood ratio test (LRT). Estimated marginal means were calculated using the selected model and pairwise comparisons were computed in the emmeans package (v1.3.5) (Lenth et al., 2018). Multiple comparisons were adjusted for each factor (in LRTs) and within each factor (for pairwise comparisons) using false discovery rate (Benjamini \& Hochberg 1995). Barplots were created using theggplots2 (Wickham, 2016) in R (v3.6.0; R Core Development Team).

\section{Results}

Factors driving microbiome alpha diversity

The microbiome community mean observed Chao1 index ranged from 240 (NIT) to 311 (CHILL), and Shannon's H diversity ranged from 3.4 (NIT) to 3.9 (YIAL, Figure 2) within crosses. Large standard errors in the alpha diversity measures were observed across all crosses in the study for Shannon and Chao1 indices. Using LRTs, no significant differences were found among crosses, among sires within crosses, or between pens within crosses (Table 1). Post-hoc pairwise t-tests analyses showed no statistically significant differences in the means between the pairs of crosses for Chao1 and Shannon indices (Table 1), corroborating the overall analysis results. Finally, the no significant population cross, sire or pen factors effects were found for either of the diversity indices (Table 1).

Factors driving global microbiome composition: Beta diversity

Overall, the first two PCoAs accounted for almost $40 \%$ of all variance in the Bray-Curtis distances across all samples. The clustering patterns revealed YIAL as an outlier cross, CHILL as intermediate, and the remaining stocks clustering more closely together on the axes (Figure 3). Using the overall PERMANOVA model, significant population effects were found in the overall microbial community structure using BrayCurtis distances $\left(p=0.001, \mathrm{R}^{2}=0.046\right)$ (Table 3). Pairwise PERMANOVA tests using Bray-Curtis distances showed that YIAL was statistically different from BQ $(p=0.0028)$, CAP $(p=0.005)$, NIT $(p=0.005)$, PUNT $(p=0.005)$, QUIN $(p=0.0065)$, and RC $(p=0.005)$ but not from CHILL $(p>0.05)$, and all other pairwise PERMANOVA comparisons showed a lack of significant pairwise differences (Supplementary Table 5). In the overall PERMANOVA model, sire effects were found to have significant effects on the microbial community $\left(p=0.002, \mathrm{R}^{2}=0.25\right.$; Table 3$)$. Furthermore, using a unique PERMANOVA model for each cross, sire effects on Bray-Curtis distances were found to be significant within CHILL and NIT ( $p=$ $0.001, \mathrm{R}^{2}=0.434$ and $p=0.006, \mathrm{R}^{2}=0.308$, respectively), but no significant sire effects were found within other crosses (Table 4). Finally, while pen effects did not contribute significantly to differences in overall microbiome community composition for Bray-Curtis distances, a significant difference was found between replicate pens for $\mathrm{RC}\left(p=0.021\right.$ and $\mathrm{R}^{2}=0.262$, Tables 4$)$.

Factors driving microbiome composition: OTU and family-level differential abundance

Overall, 1239 OTUs were found to be common to all crosses, and the largest number of unique OTUs per cross (tripletons removed) were found to be in CHILL (220), YIAL (118) and BQ (105) (Figure 4).

After correcting for multiple tests (FDR), significant cross effects were found for 13 of the 110 tested microbial OTUs in the differential abundance analysis using linear mixed effects models (Supplementary Table 2), and sire and pen effects were found for a single OTU (OTU_315506; Order: Lactobacillales ). YIAL and CHILL crosses showed the most substantial divergence at the OTU level (Figure 5). Specifically, YIAL was significantly different from at least one stock for 11 of the 13 OTUs showing significant cross effects, while additionally showing the highest or second highest relative abundance in 8 of them, after normalizing for uniform sequencing depth (Figure 5). YIAL exclusively accounted for all the pairwise differences in 3 OTUs (OTU_1061429, family: Comamonadaceae ; OTU_1085832, family:Streptococcus ; OTU_567840, family: Bradyrhizobiaceae ), and CHILL in a similar way for a single OTU (OTU_315506, family:Lactobacillales ). Interestingly, each of RC and NIT additionally showed significant pairwise comparison differences for a single OTU (OTU_145914, family: Mycoplasmataceae ; OTU_NF_92, family:Sphingomonadaceae ). A single 
OTU did not occur in CAP: OTU_315506 (Order: Lactobacillales ).

Using linear mixed effects model to test for hybrid cross, sire and pen effects, significant cross differences were found for seven (out of 44) tested taxonomic families (Supplementary Table 3), pen effects were found for 3 taxonomic families (Streptococcaceae, Carnobacteriaceae, and Lactobacillaceae) and sire effects were found for a single taxonomic family (Bacillaceae). Similar to OTU-level differences, YIAL and CHILL showed the greatest divergence in pairwise comparisons across all taxonomic families (Figure 6). Substantial divergence was exhibited by YIAL in 4 taxonomic families (Bacillaceae, Streptococcaceae, Chitinophagaceae , and Mycobacteriaceae) and by CHILL in 2 taxonomic families (Leuconostocaceae, Lactobacillaceae ), and by both crosses in Carnobacteriaceae (Figure 6).

\section{Discussion}

Host effects on the microbiome are less commonly reported at the among-population than within-population level, since many studies are based on single populations, albeit often involving various treatments (see Tables $2 \&$ 3, Ghanbari et al., 2015). Here, we provide evidence for significant cross effects reflective of strong interpopulation genetic divergence effects, and significant sire effects indicative of additive genetics effects acting within populations on the composition of the microbiome at the community (alpha- and beta-diversity) and finer (OTUs and taxonomic families) levels of the microbiome. In addition, we also found significant, but small and rare, pen effects that reflect environmental effects. The pen effects were not expected as the replicate pens were designed to be as similar as possible (size, water quality, feeding regime, etc.); however, these differences are likely due to the generally reported high magnitude of environmental drivers on the microbiome (Wu et al.,2013; Goodrich et al., 2014a; Sullam et al., 2015; Rothschild et al., 2018). Based on the published literature for fish, we expected to find among-population gut microbiome differences, indicative of previously reported host genetic divergence effects and the known role of the microbiome in assisting the hosts to cope with their environment (Sullam et al., 2015; Webster et al.,2018). As for sire effects, the differences observed in the microbiome phenotypic outcome were not expected due to the generally low heritability reported for humans (Yatsunenko et al., 2012; Kurilshikov, Wijmenga, Fu \& Zhernakova, 2017; Rothschild et al.,2018; but see Goodrich et al., 2014b), although this is the first report of additive genetic variation studies in fish microbiome composition. Given that all of the offspring in this study were reared in a common environment, from a common dam, two evolutionary processes may explain the among-stock differences found among the microbiomes: genetic drift and natural selection. Overall, the pattern of observed microbiome-based phenotypic differences among population crosses are consistent with patterns of host-microbiome co-divergence (Sullamet al., 2015), while the pattern of within-population additive genetic variance may have been shaped by population-specific selection pressures resulting from local stressors experienced in their native habitats (Savolainen, Lascoux \& Merilä, 2013).

We found significant and consistent population cross effects on the composition of the microbiome, which reflect, primarily, among-population effects. Interpopulation differences and effects of origin (wild vs. hatchery) on the gut microbiome were recently reported using three hatchery-reared and four wild Atlantic salmon populations, showing substantial differences in the overall microbiome composition (beta diversity) and the core microbiome (Webster et al., 2018). While the study did find larger differences among the genetically more divergent populations (based on microsatellite marker genotypes), it lacked the power to control for environmental effects, and diet was suspected to be a major factor driving the variation in composition of the microbiome among the studied populations (Webster et al.,2018). It is critical to note in the breeding design used in this study, emphasis was placed on studying the contribution of cross and sire effects while controlling for maternal effects on growth (Semeniuket al., 2019). In our breeding design, we were able to estimate additive effects while virtually eliminating the potential for maternal effects. Maternal effects are composed of environmental and dam effects (Aykanat, Bryden \& Heath 2012a) and are known to contribute substantially to among-population phenotypic variation in Chinook salmon for life history and fitness-related traits (Aykanat, Heath, Dixon \& Heath 2012b). By using a common dam in our breeding design, we eliminated the potential for these maternal effects, thereby potentially limiting the detection of the maternal adaptive microbiome variance, such as those relating to immunity in Chinook salmon (Aykanat et al., 2012a). 
While consistent beta diversity population effects were observed, alpha diversity effects were not, despite other studies that did report them (Dehler, Secombes \& Martin, 2017; Reveco et al., 2014; Websteret al., 2018). This might be due to the common and controlled pens. In this study, the magnitude of population cross effects on the microbiome varied in a consistent manner across analytical approaches: beta diversity and variation at the individual OTU or taxonomic family levels showed that the fully-domesticated cross, YIAL, exhibited the most divergent microbiome characteristics relative to all the hybrid crosses. It is likely that these differences derive from strong domestication selective pressures experienced within the YIAL production stock, perhaps driving rapid divergence in both the host and gut microbiome community. Interestingly, YIAL was not found to be significantly different in microbial community structure from CHILL, indicating that CHILL possess an intermediate microbiome structure. Although we found no differences in pairwise comparisons of microbial community structure level for CHILL versus the other hybrid cross stocks, they did approach significance for some populations (BQ, NIT, and PUNT), which suggests that functional differences may be present, but were undetectable given the statistical power of this study. Using the same study system and hybrid crosses, the CHILL hybrid cross was shown to vary from the other crosses in related studies. For example, in a study designed to detect gene expression differences among and within the hybrid crosses, CHILL exhibited a marked difference in gene transcription profile relative to the other hybrid cross stocks (including YIAL) consistent with the observe divergence pattern in this study (Toews, Wellband, Dixon \& Heath, 2019). Furthermore, over the entire production period, CHILL was found to exhibit the lowest survival relative to the other crosses (Semeniuk et al., 2019). Local adaptation occurs when there is strong selection and limited drift effects (Yeaman and Otto, 2012). While large floods were experienced in the Chilliwack River (CHILL) between the years of 1952 and 1980 (Ham, 1996), conclusions about possible drift genetic (e.g. bottleneck events) are difficult to make, as the impact of those floods on Chilliwack River Chinook salmon stocks is unknown (Bradford, 1995). While we cannot rule out genetic drift acting to differentiate our study populations for gut microbiome composition (e.g., Whitehead, 2012), the patterns we observed at the OTU and bacterial family levels - which may involve crucial symbiotic roles - suggest divergent selection effects (Kawecki and Ebert, 2004). Therefore, our individual taxon analyses (at the OTU and taxonomic family levels) allowed us to explore potentially functional patterns of differences among the hybrid cross stocks. With some exceptions, YIAL and CHILL harboured significantly lower counts of several lactic acid bacteria (LABs), thought to contribute favourably to host health in fish (Ingerslev et al., 2014; He, Chaganti \& Heath, 2018). If higher LAB abundance is indeed adaptive, this suggests that lower LAB levels may account for the low survival observed in CHILL (Semeniuk et al., 2019). However, it also leaves the higher levels of Lactobacillales and Lactobacillaceae in CHILL unexplained. Interestingly, CHILL also showed high levels of the pathogen Micrococcus luteus, which has been associated with health disorders in rainbow trout (Austin and Stobie, 1992; Pekalaet al., 2018) and brown trout (Salmo trutta) (Pekalaet al., 2018). Together, these patterns suggest that individuals from our hybrid population crosses may experience reduced resistance, potentially due to hybrid breakdown, wherein a farmed population outbreeding with a wild counterpart results in reduced performance (Edmands, 1999; Lehnert, Love, Pitcher, Higgs \& Heath, 2014). Furthermore, given that YIAL exhibited intermediate cumulative survival (Semeniuk et al., 2019), this begs the question of whether YIAL has adapted to survive in this environment through means other than accumulating beneficial bacteria through its multi-generational domesticated rearing. Finally, YIAL and CHILL harboured higher counts of OTUs classified as Comamonadaceae or Bradyrhizobiaceae, which commonly exhibit biochemical and ecological versatility (de Souza, Carrareto Alves, de Mello Varani \& de Macedo Lemos, 2014; Willems, 2014). While not conclusive, these results point towards non-neutral co-divergence in host genetic architecture and microbiome community structure. Further work is needed to characterize the effects culminating in the formation of divergent microbiome community compositions among population crosses.

Significant within-population family differences, or sire effects, allowed us to estimate additive genetic variation effects. Additive genetic variation is a critical component of the overall genetic architecture for any trait, and it defines the scope for traditional evolutionary response to selection (Gjedrem, 1983; Garcia de Leanizet al., 2007; Visscher et al., 2008; van Open, Oliver, Putnam \& Gates, 2015). Within-population microbiome variation was found among unrelated families of rainbow trout (Naverrete et al.,2012), but esti- 
mates of additive genetic variation in fish gut microbiome studies are lacking. This study presents the first report of additive genetic variance effects on the microbiome composition in fish. While the contribution of additive genetic effects to the composition of the gut microbiome in fish has not been studied, efforts have been made to quantify it in human studies, mainly showing that the microbiome generally exhibits low heritability (Yatsunenko et al., 2012; Kurilshikov et al., 2017; Rothschild et al., 2018). In the breeding design used in this study, variation among sires within stocks were estimated using half-sibling families as a measure of additive genetic variance, since a common egg source (i.e. highly inbred females combined) was used for all stocks. Given this breeding design and previous reports of low additive genetic variance, we expected that no additive genetic effects would be observed in this study. Despite that, we found consistent additive genetic effects on the on the microbiome composition at the beta-diversity level of the microbiome. Interestingly, significant additive genetic effects were found to be stock-specific (e.g., CHILL and NIT), perhaps reflecting lower selection pressures on the microbiome experienced in those populations. A previous study showed that microbial quantitative trait loci (mbQTLs) interact with host immunity to shape the gut microbiome in humans (Kurilshikovet al., 2017). Additionally, MHC class II complex genotypes contribute to the regulation of the microbiome composition among hosts in a sex dependent manner in three-spine stickleback (Gasterosteus aculeatus ) (Bolnick et al., 2014). Although maternal vertical transmission is unlikely to be contributing to our additive genetic variation estimates, the role of paternal vertical transmission or other epigenetic effects on offspring microbiome composition have not yet been investigated. Variation in underlying genetic architecture (specifically additive genetic variance) among populations is critical to predict a population's response to selection and are a requisite for selective commercial (e.g. aquaculture) and non-commercial (e.g. conservation and restoration) breeding applications (Gjedrem, 1983; Visscher et al., 2008; van Open et al., 2015).

Pairs of replicate net pens for each population were included to allow the partitioning of possible environmental effects; however, our use of common rearing environments and matched net pens made strong environmental effects on gut microbiome unlikely. Nevertheless, replicate pens effects were found to be significant for microbial community composition for the RC hybrid-cross, and in the differential abundance analysis at a single OTU (OTU_315506, orderLactobacillales ) and three taxonomic families (Carnobacteriaceae, Lactobacillaceae andLeuconostocaceae), suggesting that some form of environmental effects across pens contributed to microbiome variation at the specific taxon level. These environmental effects may be explained by fine-scale environmental heterogeneity. Such effects can drive subtle phenotypic differences, often complicating the study of local adaptation, or genetics, in host-microbe systems (Kaltz \& Shykoff, 1998, Savolainenet al., 2013). Furthermore, we suspect that uncontrollable variation in social interactions among individuals may exist within pens (Gilmour et al., 2005), and drive microbiome differences between replicates. This emphasizes the challenge in minimizing the effect of the environmental factors driving the gut microbiome, which have been shown to dominate host-related factors in humans (Wu et al.,2013; Rothschild et al., 2018).

In conclusion, our study shows a rarely reported pattern of population-level variation in the gut microbiome community in fish. Such a pattern is consistent with local adaptation, perhaps due to strong selection associated with seven generations of domestication combined with local selection forces acting to create divergent microbiome community compositions. Inter-population effects were the largest and most consistent drivers of gut microbiome variation among the hybrid cross stocks. Additive genetic variance and environmental effects contributed to variation at different hierarchical levels of the microbiome, with additive genetic contributing at the overall microbiome composition more strongly than pen effects. Microbiome OTU and taxonomic family effects were found to be population-specific, further supporting the role of local population effects driving microbiome structure, despite rearing in a common environment with a common dam. Our results highlight the importance of preserving genetic variation in Chinook salmon to respond to environmental heterogeneity especially in the face of oceanic climate changes and habitat degradation from urban development and anthropogenic practices.

\section{Acknowledgements:}


This study is part of a larger cooperative project that involved the dedicated work of many researchers, and for their assistance in the field, we would like to acknowledge the following: N. Bernier, J. Camaj, E. Deck , A. Forest, X. He, V.A. Heath, K. O'Hagan-Wang, J. Drown, J.W. Heath, V.A. Heath and J. Smit We would like to thank Yellow Island Aquaculture, Ltd. for their invaluable logistical, financial, and technical support.

This study was funded by a Natural Science and Engineering Research Council (NSERC) of Canada Strategic Partnership Grant, and an NSERC Discovery Grant for the last author.

\section{Data Accessibility Statement}

Raw sequences that support the findings of this study are publically available from the National Center for Biotechnology Information, under BioProject ID PRJNA628094 and Submission ID SUB7309667.

\section{Author Contributions}

D.D.H. conceived and planned the experiments. D.D.H. and S.R.C. carried out field work. S.R.C contributed to the wet laboratory sample preparation, however, most of the wet lab work was done by M.Z. All authors contributed to selecting the models and the computational framework for the data analysis and interpreting the results. M.Z. took the lead in performing the research, analyzing data and writing the manuscript. All authors provided critical feedback and helped shape the research, analysis and manuscript.

\section{References}

Akaike, H. (1973). Maximum likelihood identification of Gaussian autoregressive moving average models. Biometrika, 60(2), 255-265.

Anderson, M. J. (2001). A new method for non-parametric multivariate analysis of variance. Austral ecology , $26(1), 32-46$.

Antychowicz, J., \& Lipiec, M. (2006). Mycobacteriaceae pathogenic for fish and human. Medycyna Weterynaryjna , 62 (7), 731-735.

Austin, B., \& Stobie, M. (1992). Recovery of Micrococcus luteus and presumptive Planococcus sp. from moribund fish during an outbreak of rainbow trout, Oncorhynchus mykiss (Walbaum), fry syndrome in England. Journal of Fish Diseases , 15 (2), 203-206.

Austin, B., Austin, D. A., Austin, B., \& Austin, D. A. (2012). Bacterial fish pathogens (Vol. 481, p. 482). Dordrecht, The Netherlands: Springer.

Aykanat, T., Heath, J. W., Dixon, B., \& Heath, D. D. (2012a). Additive, non-additive and maternal effects of cytokine transcription in response to immunostimulation with Vibrio vaccine in Chinook salmon (Oncorhynchus tshawytscha). Immunogenetics , 64 (9), 691-703.

Aykanat, T., Bryden, C. A., \& Heath, D. D. (2012b). Sex-biased genetic component distribution among populations: additive genetic and maternal contributions to phenotypic differences among populations of Chinook salmon. Journal of evolutionary biology , 25 (4), 682-690.

Bledsoe, J. W., Peterson, B. C., Swanson, K. S., \& Small, B. C. (2016). Ontogenetic characterization of the intestinal microbiota of channel catfish through $16 \mathrm{~S}$ rRNA gene sequencing reveals insights on temporal shifts and the influence of environmental microbes. PloS one , 11 (11).

Bolnick, D. I., Snowberg, L. K., Hirsch, P. E., Lauber, C. L., Org, E., Parks, B., . . \& Svanbäck, R. (2014). Individual diet has sex-dependent effects on vertebrate gut microbiota. Nature communications , 5 , 4500.

Booman, M., Forster, I., Vederas, J. C., Groman, D. B., \& Jones, S. R. (2018). Soybean meal-induced enteritis in Atlantic salmon (Salmo salar) and Chinook salmon (Oncorhynchus tshawytscha) but not in pink salmon (O. gorbuscha ). Aquaculture, 483 , 238-243.

Bradford, M. J. (1995). Comparative review of Pacific salmon survival rates. Canadian Journal of Fisheries and Aquatic Sciences, 52 (6), 1327-1338. 
Brooks, M. E., Kristensen, K., van Benthem, K. J., Magnusson, A., Berg, C. W., Nielsen, A., .. \& Bolker, B. M. (2017). Modeling zero-inflated count data with glmmTMB. BioRxiv , 132753.

Caporaso, J. G., Kuczynski, J., Stombaugh, J., Bittinger, K., Bushman, F. D., Costello, E. K., .. \& \& Huttley, G. A. (2010). QIIME allows analysis of high-throughput community sequencing data. Nature methods, 7 (5), 335 .

Caporaso, J. G., Bittinger, K., Bushman, F. D., DeSantis, T. Z., Andersen, G. L., \& Knight, R. (2010). PyNAST: a flexible tool for aligning sequences to a template alignment. Bioinformatics , 26 (2), 266-267.

Ciric, M., Waite, D., Draper, J., \& Jones, J. B. (2018). Characterisation of gut microbiota of farmed Chinook salmon using metabarcoding. bioRxiv: The Preprint server for Biology .

Ciric, M., Waite, D., Draper, J., \& Jones, J. B. (2019). Characterization of mid-intestinal microbiota of farmed Chinook salmon using 16S rRNA gene metabarcoding. Archives of Biological Sciences , 71 (4), 577-587.

de Souza, J. M., Carrareto Alves, L. M., de Mello Varani, A., \& de Macedo Lemos, E. G. (2014). The family Bradyrhizobiaceae. The Prokaryotes, 135-154.

Dehler, C. E., Secombes, C. J., \& Martin, S. A. (2017). Environmental and physiological factors shape the gut microbiota of Atlantic salmon parr (Salmo salar L.). Aquaculture , 467, 149-157.

DFO. 2018 Canadian Aquaculture Production Statistics | Fisheries and Oceans Canada , 2018, https://www.dfompo.gc.ca/stats/aqua/aqua18-eng.htm

Eschmeyer, W. N., \& Fong, J. D. (2015). Species by family/subfamily in the Catalog of Fishes. San Francisco, CA: California Academy of Sciences .

Edgar, R. C. (2010). Search and clustering orders of magnitude faster than BLAST. Bioinformatics , 26 (19), 2460-2461.

Edmands, S. (1999). Heterosis and outbreeding depression in interpopulation crosses spanning a wide range of divergence. Evolution , 53 (6), 1757-1768.

Foster, K. R., Schluter, J., Coyte, K. Z., \& Rakoff-Nahoum, S. (2017). The evolution of the host microbiome as an ecosystem on a leash. Nature, 548 (7665), 43-51.

Fraser, D. J., Weir, L. K., Bernatchez, L., Hansen, M. M., \& Taylor, E. B. (2011). Extent and scale of local adaptation in salmonid fishes: review and meta-analysis. Heredity , 106 (3), 404-420.

Garcia de Leaniz, C., Fleming, I. A., Einum, S., Verspoor, E., Jordan, W. C., Consuegra, S., ... \& Webb, J. H. (2007). A critical review of adaptive genetic variation in Atlantic salmon: implications for conservation. Biological reviews, 82 (2), 173-211.

Ghanbari, M., Kneifel, W., \& Domig, K. J. (2015). A new view of the fish gut microbiome: advances from next-generation sequencing. Aquaculture , 448, 464-475.

Gillespie, J. H. (2004). Population genetics: a concise guide. JHU Press.

Gjedrem, T. (1983). Genetic variation in quantitative traits and selective breeding in fish and shellfish. Aquaculture, 33 (1-4), 51-72.

Goodrich, J. K., Di Rienzi, S. C., Poole, A. C., Koren, O., Walters, W. A., Caporaso, J. G., .. \& Ley, R. E. (2014a). Conducting a microbiome study. Cell , 158 (2), 250-262.

Goodrich, J. K., Waters, J. L., Poole, A. C., Sutter, J. L., Koren, O., Blekhman, R., ... \& Spector, T. D. (2014b). Human genetics shape the gut microbiome. Cell , 159 (4), 789-799.

Ham, D. G. (1996). Patterns of channel change on Chilliwack River, British Columbia (Doctoral dissertation, University of British Columbia). 
Hammer, Ø., Harper, D. A., \& Ryan, P. D. (2001). PAST : Paleontological statistics software package for education and data analysis. Palaeontologia electronica , 4 (1), 9.

He, X., Chaganti, S. R., \& Heath, D. D. (2018). Population-specific responses to interspecific competition in the gut microbiota of two Atlantic Salmon (Salmo salar ) populations. Microbial ecology, 75 (1), 140-151.

Heath, D. D., Fox, C. W., \& Heath, J. W. (1999). Maternal effects on offspring size: variation through early development of chinook salmon. Evolution , 53 (5), 1605-1611.

Hilderbrand, G. V., Farley, S. D., Schwartz, C. C., \& Robbins, C. T. (2004). Importance of salmon to wildlife: implications for integrated management. Ursus , 15 (1), 1-9.

Ingerslev, H. C., Strube, M. L., von Gersdorff Jørgensen, L., Dalsgaard, I., Boye, M., \& Madsen, L. (2014). Diet type dictates the gut microbiota and the immune response against Yersinia ruckeri in rainbow trout (Oncorhynchus mykiss ). Fish 8 shellfish immunology , 40 (2), 624-633.

Kaltz, O., \& Shykoff, J. A. (1998). Local adaptation in host-parasite systems. Heredity , 81 (4), 361-370.

Kawecki, T. J., \& Ebert, D. (2004). Conceptual issues in local adaptation. Ecology letters , 7 (12), 1225-1241.

Kim, J. E., Withler, R. E., Ritland, C., \& Cheng, K. M. (2004). Genetic variation within and between domesticated chinook salmon, Oncorhynchus tshawytscha, strains and their progenitor populations. Environmental biology of fishes, 69(1-4), 371-378.

Koskella, B., Hall, L. J., \& Metcalf, C. J. E. (2017). The microbiome beyond the horizon of ecological and evolutionary theory. Nature ecology $\&$ evolution , 1 (11), 1606-1615.

Kurilshikov, A., Wijmenga, C., Fu, J., \& Zhernakova, A. (2017). Host genetics and gut microbiome: challenges and perspectives. Trends in immunology , 38 (9), 633-647.

Larsen, A. M., Mohammed, H. H., \& Arias, C. R. (2014). Characterization of the gut microbiota of three commercially valuable warmwater fish species. Journal of applied microbiology, 116 (6), 1396-1404.

Lehnert, S. J., Love, O. P., Pitcher, T. E., Higgs, D. M., \& Heath, D. D. (2014). Multigenerational outbreeding effects in Chinook salmon (Oncorhynchus tshawytscha ). Genetica , 142 (4), 281-293.

Lenth, R., Singmann, H., Love, J, Buerkner, P., \& Herve, P. "Emmeans: Estimated marginal means, aka least-squares means." $R$ package version 1.1 (2018).

Ley, R. E., Hamady, M., Lozupone, C., Turnbaugh, P. J., Ramey, R. R., Bircher, J. S., .. \& \& Gordon, J. I. (2008). Evolution of mammals and their gut microbes. Science, 320 (5883), 1647-1651.

Lex, A., Gehlenborg, N., Strobelt, H., Vuillemot, R., \& Pfister, H. (2014). UpSet : visualization of intersecting sets. IEEE transactions on visualization and computer graphics , 20 (12), 1983-1992.

Li, T., Long, M., Gatesoupe, F. J., Zhang, Q., Li, A., \& Gong, X. (2015). Comparative analysis of the intestinal bacterial communities in different species of carp by pyrosequencing. Microbial ecology , 69 (1), $25-36$.

Llewellyn, M. S., Boutin, S., Hoseinifar, S. H., \& Derome, N. (2014). Teleost microbiomes: the state of the art in their characterization, manipulation and importance in aquaculture and fisheries. Frontiers in microbiology , 5, 207.

Llewellyn, M. S., McGinnity, P., Dionne, M., Letourneau, J., Thonier, F., Carvalho, G. R., .. \& Derome, N. (2016). The biogeography of the Atlantic salmon (Salmo salar) gut microbiome. The ISME journal , 10 (5), 1280-1284.

Lynch M, Walsh B. Genetics and analysis of quantitative traits. Sunderland, MA: Sinauer; 1998 Jan.

Magnusson, A., Skaug, H., Nielsen, A., Berg, C., Kristensen, K., Maechler, M., .. \& Brooks, M. M. (2017). Package 'glmmTMB'. R Package Version 0.2.0. 
Navarrete, P., Magne, F., Araneda, C., Fuentes, P., Barros, L., Opazo, R., .. \& Romero, J. (2012). PCRTTGE analysis of $16 \mathrm{~S}$ rRNA from rainbow trout (Oncorhynchus mykiss) gut microbiota reveals host-specific communities of active bacteria. PloS one, 7 (2).

Nelson, J. S., Grande, T. C., \& Wilson, M. V. (2016). Fishes of the World . John Wiley \& Sons.

NOAA. "Chinook Salmon - Protected." NOAA Fisheries, 2018, www.fisheries.noaa.gov/species/chinooksalmon-protected.

O'Brien, P. A., Webster, N. S., Miller, D. J., \& Bourne, D. G. (2019). Host-microbe coevolution: applying evidence from model systems to complex marine invertebrate holobionts. MBio , 10 (1), e02241-18.

Ohlberger, J., Ward, E. J., Schindler, D. E., \& Lewis, B. (2018). Demographic changes in Chinook salmon across the Northeast Pacific Ocean. Fish and Fisheries , 19 (3), 533-546.

Oksanen, J., Blanchet, F. G., Kindt, R., Legendre, P., Minchin, P. R., O'hara, R. B., .. \& Oksanen, M. J. (2013). Package 'vegan'. Community ecology package, version, 2 (9), 1-295.

Parshukov, A. N., Kashinskaya, E. N., Simonov, E. P., Hlunov, O. V., Izvekova, G. I., Andree, K. B., \& Solovyev, M. M. (2019). Variations of the intestinal gut microbiota of farmed rainbow trout, Oncorhynchus mykiss (Walbaum), depending on the infection status of the fish. Journal of applied microbiology, 127(2), 379-395.

Paulson, J. N., Stine, O. C., Bravo, H. C., \& Pop, M. (2013). Differential abundance analysis for microbial marker-gene surveys. Nature methods, 10 (12), 1200.

Pekala, A., Paździor, E., Antychowicz, J., Bernad, A., Głowacka, H., Wiecek, B., \& Niemczuk, W. (2018). Kocuria rhizophila and Micrococcus luteus as emerging opportunist pathogens in brown trout (Salmo trutta Linnaeus, 1758) and rainbow trout (Oncorhynchus mykiss Walbaum, 1792). Aquaculture, 486, 285-289.

Quinn, T. P. (2018). The behavior and ecology of Pacific salmon and trout . University of Washington press.

Roeselers, G., Mittge, E. K., Stephens, W. Z., Parichy, D. M., Cavanaugh, C. M., Guillemin, K., \& Rawls, J. F. (2011). Evidence for a core gut microbiota in the zebrafish. The ISME journal , 5 (10), 1595-1608.

Romero, J., Ring $\varnothing$, E., \& Merrifield, D. L. (2014). The gut microbiota of fish. Aquaculture nutrition: Gut health, probiotics and prebiotics, 75-100.

Rothschild, D., Weissbrod, O., Barkan, E., Kurilshikov, A., Korem, T., Zeevi, D., .. \& Shilo, S. (2018). Environment dominates over host genetics in shaping human gut microbiota. Nature , 555 (7695), 210-215.

Rounsefell, G. A. (1958). Anadromy in North American Salmonidae. US Government Printing Office.

Savolainen, O., Lascoux, M., \& Merilä, J. (2013). Ecological genomics of local adaptation. Nature Reviews Genetics , 14 (11), 807-820.

Semeniuk, C. A., Capelle, P. M., Dender, M. G., Devlin, R., Dixon, B., Drown, J., .. \& Lehnert, S. J. (2019). Domestic-wild hybridization to improve aquaculture performance in Chinook salmon. Aquaculture , 511, 734255 .

Spor, A., Koren, O., \& Ley, R. (2011). Unravelling the effects of the environment and host genotype on the gut microbiome. Nature Reviews Microbiology , 9 (4), 279-290.

Sullam, K. E., Rubin, B. E., Dalton, C. M., Kilham, S. S., Flecker, A. S., \& Russell, J. A. (2015). Divergence across diet, time and populations rules out parallel evolution in the gut microbiomes of Trinidadian guppies. The ISME journal, 9(7), 1508-1522.

Team, R. C. (2000). R language definition. Vienna, Austria: R foundation for statistical computing. 
Toews, S. D., Wellband, K. W., Dixon, B., \& Heath, D. D. (2019). Variation in juvenile Chinook salmon (Oncorhynchus tshawytscha) transcription profiles among and within eight population crosses from British Columbia, Canada. Molecular ecology , 28 (8), 1890-1903.

van Oppen, M. J., Oliver, J. K., Putnam, H. M., \& Gates, R. D. (2015). Building coral reef resilience through assisted evolution. Proceedings of the National Academy of Sciences , 112 (8), 2307-2313.

Visscher, P. M., Hill, W. G., \& Wray, N. R. (2008). Heritability in the genomics era-concepts and misconceptions. Nature reviews genetics, 9 (4), 255-266.

Waples, R. S., Naish, K. A., \& Primmer, C. R. (2019). Conservation and Management of Salmon in the Age of Genomics. Annual review of animal biosciences , 8 .

Webster, T. M. U., Consuegra, S., Hitchings, M., \& de Leaniz, C. G. (2018). Interpopulation variation in the Atlantic salmon microbiome reflects environmental and genetic diversity. Appl. Environ. Microbiol. , 84 (16), e00691-18.

Weiss, S., Xu, Z. Z., Peddada, S., Amir, A., Bittinger, K., Gonzalez, A., .. \& Hyde, E. R. (2017). Normalization and microbial differential abundance strategies depend upon data characteristics. Microbiome , 5 (1), 27.

Whitehead, A. (2012). Comparative genomics in ecological physiology: toward a more nuanced understanding of acclimation and adaptation. Journal of Experimental Biology , 215 (6), 884-891.

Wickham, H. (2016). ggplot2: elegant graphics for data analysis . Springer.

Willems, A. (2014). The family Comamonadaceae. The prokaryotes: Alphaproteobacteria and Betaproteobacteria. The Prokaryotes. Berlin: Springer .

Wong, S., \& Rawls, J. F. (2012). Intestinal microbiota composition in fishes is influenced by host ecology and environment. Molecular ecology, 21 (13), 3100-3102.

Wong, S., Waldrop, T., Summerfelt, S., Davidson, J., Barrows, F., Kenney, P. B., .. \& Good, C. (2013). Aquacultured rainbow trout (Oncorhynchus mykiss) possess a large core intestinal microbiota that is resistant to variation in diet and rearing density. Appl. Environ. Microbiol. , 79 (16), 4974-4984.

Wu, S. G., Tian, J. Y., Gatesoupe, F. J., Li, W. X., Zou, H., Yang, B. J., \& Wang, G. T. (2013). Intestinal microbiota of gibel carp (Carassius auratus gibelio ) and its origin as revealed by 454 pyrosequencing. World Journal of Microbiology and Biotechnology , 29 (9), 1585-1595.

Xu, L., Paterson, A. D., Turpin, W., \& Xu, W. (2015). Assessment and selection of competing models for zero-inflated microbiome data. PloS one, 10 (7).

Yatsunenko, T., Rey, F. E., Manary, M. J., Trehan, I., Dominguez-Bello, M. G., Contreras, M., ... \& Heath, A. C. (2012). Human gut microbiome viewed across age and geography. nature , 486 (7402), 222-227.

Yeaman, S., \& Otto, S. P. (2011). Establishment and maintenance of adaptive genetic divergence under migration, selection, and drift. Evolution: International Journal of Organic Evolution, 65 (7), 2123-2129.

Tables

Table 1: Alpha diversity analysis results using LMMs. The likelihood ratio test was used to calculate the significance of the differences for each alpha diversity metric. (Significance codes: ${ }^{* * *} 0.001{ }^{* * *} 0.01$ “*, 0.05 )

\begin{tabular}{lllll}
\hline Response & Factor & ChiSq & df & P-value \\
\hline Shannon & Cross & 7.45 & 7 & 0.38 \\
& Pen & 0 & 2 & 1 \\
& Sire & 0.73 & 2 & 0.70 \\
Chao1 & Cross & 14.44 & 7 & 0.065
\end{tabular}




\begin{tabular}{lllll}
\hline Response & Factor & ChiSq & df & P-value \\
\hline & Pen & 0 & 2 & 1 \\
& Sire & 0.33 & 2 & 0.88 \\
\hline
\end{tabular}

Table 2. Results of overall PERMANOVA analysis using the Bray-Curtis dissimilarity distances. Sire and Pen factors are nested within Cross. Significance codes: ${ }^{* * *}, 0.001{ }^{* * *} 0.01{ }^{* *}, 0.05$ )

\begin{tabular}{llllllll}
\hline Factor & Df & Sums of Squares & Mean Squares & \% Variation & F-Model & $\mathrm{R}^{2}$ & P-value \\
\hline Cross & 7 & 3.72 & 0.53 & 37.69 & 1.97 & 0.05 & $0.001^{* * *}$ \\
Sire & 59 & 19.91 & 0.34 & 23.95 & 1.25 & 0.25 & $0.002^{* *}$ \\
Pen & 8 & 2.17 & 0.27 & 19.24 & 1.01 & 0.03 & 0.443 \\
Residuals & 203 & 54.68 & 0.27 & 19.12 & & 0.68 & \\
Total & 277 & 80.47 & 1.41 & 100.00 & & 1.00 & \\
\hline
\end{tabular}

Table 3. Pairwise PERMANOVA comparisons (BH-corrected p-values) based on Bray-Curtis. The number of permutations used in the analysis was 9999. P-values are corrected to two significant figures. Significance codes: $\left.{ }^{* * *}, 0.001{ }^{* * *} 0.01{ }^{* *}, 0.05\right)$

\begin{tabular}{llllllll}
\hline & BQ & CAP & CHILL & NIT & PUNT & QUIN & RC \\
\hline CAP & 0.75 & & & & & & \\
CHILL & 0.067 & 0.08 & & & & & \\
NIT & 0.52 & 0.79 & 0.067 & & & & \\
PUNT & 0.71 & 0.76 & 0.067 & 0.75 & & & \\
QUIN & 0.31 & 0.54 & 0.076 & 0.79 & 0.50 & & \\
RC & 0.75 & 0.87 & 0.11 & 0.76 & 0.70 & 0.75 & \\
YIAL & $0.0028^{* *}$ & $0.005^{* *}$ & 0.097 & $0.005^{* *}$ & $0.005^{* *}$ & $0.0065^{* *}$ & $0.005^{* *}$ \\
\hline
\end{tabular}

Table 4. Population cross-specific results of nested-PERMANOVA analysis using the Bray-Curtis distance.

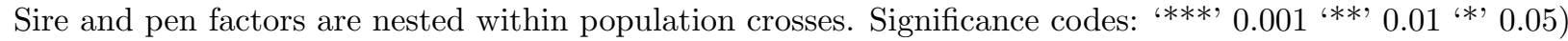

\begin{tabular}{|c|c|c|c|c|c|c|c|}
\hline $\begin{array}{l}\text { Population } \\
\text { Cross }\end{array}$ & Factor & Df & $\begin{array}{l}\text { Sum of } \\
\text { Squares }\end{array}$ & $\begin{array}{l}\text { Mean } \\
\text { Squares }\end{array}$ & F-Model & $\mathrm{R}^{2}$ & $\operatorname{Pr}(>F)$ \\
\hline \multirow[t]{4}{*}{$\mathrm{BQ}$} & Sire & 7 & 2.26 & 0.32 & 1.16 & 0.20 & 0.20 \\
\hline & Pen & 7 & 1.96 & 0.28 & 1.00 & 0.18 & 0.48 \\
\hline & Residuals & 25 & 6.98 & 0.28 & & 0.62 & \\
\hline & Total & 39 & 11.21 & 0.88 & & 1.00 & \\
\hline \multirow[t]{4}{*}{ CAP } & Sire & 7 & 2.24 & 0.32 & 1.05 & 0.24 & 0.37 \\
\hline & Pen & 6 & 1.13 & 0.19 & 0.61 & 0.12 & 1.00 \\
\hline & Residuals & 19 & 5.80 & 0.31 & & 0.63 & \\
\hline & Total & 32 & 9.17 & 0.81 & & 1.00 & \\
\hline \multirow[t]{4}{*}{ CHILL } & Sire & 7 & 3.15 & 0.45 & 2.01 & 0.43 & $0.001^{* * *}$ \\
\hline & Pen & 6 & 1.21 & 0.20 & 0.90 & 0.17 & 0.73 \\
\hline & Residuals & 13 & 2.91 & 0.22 & & 0.40 & \\
\hline & Total & 26 & 7.27 & 0.87 & & 1.00 & \\
\hline \multirow[t]{2}{*}{ NIT } & Sire & 8 & 3.10 & 0.39 & 1.65 & 0.31 & $0.006^{* *}$ \\
\hline & Pen & 6 & 1.80 & 0.30 & 1.27 & 0.18 & 0.11 \\
\hline
\end{tabular}




\begin{tabular}{|c|c|c|c|c|c|c|c|}
\hline $\begin{array}{l}\text { Population } \\
\text { Cross }\end{array}$ & Factor & Df & $\begin{array}{l}\text { Sum of } \\
\text { Squares }\end{array}$ & $\begin{array}{l}\text { Mean } \\
\text { Squares }\end{array}$ & F-Model & $\mathrm{R}^{2}$ & $\operatorname{Pr}(>\mathrm{F})$ \\
\hline & Residuals & 22 & 5.18 & 0.24 & & 0.51 & \\
\hline & Total & 36 & 10.07 & 0.92 & & 1.00 & \\
\hline \multirow[t]{4}{*}{ PUNT } & Sire & 8 & 2.45 & 0.31 & 1.14 & 0.25 & 0.21 \\
\hline & Pen & 7 & 2.16 & 0.31 & 1.15 & 0.22 & 0.21 \\
\hline & Residuals & 19 & 5.11 & 0.27 & & 0.53 & \\
\hline & Total & 34 & 9.73 & 0.88 & & 1.00 & \\
\hline \multirow[t]{4}{*}{ QUIN } & Sire & 8 & 2.71 & 0.34 & 1.22 & 0.30 & 0.14 \\
\hline & Pen & 7 & 1.69 & 0.24 & 0.87 & 0.19 & 0.75 \\
\hline & Residuals & 17 & 4.71 & 0.28 & & 0.52 & \\
\hline & Total & 32 & 9.11 & 0.86 & & 1.00 & \\
\hline \multirow[t]{4}{*}{$\mathrm{RC}$} & Sire & 7 & 2.07 & 0.30 & 1.21 & 0.24 & 0.18 \\
\hline & Pen & 6 & 2.30 & 0.38 & 1.56 & 0.26 & $0.021^{*}$ \\
\hline & Residuals & 18 & 4.41 & 0.25 & & 0.50 & \\
\hline & Total & 31 & 8.78 & 0.92 & & 1.00 & \\
\hline \multirow[t]{4}{*}{ YIAL } & Sire & 7 & 1.91 & 0.27 & 0.97 & 0.17 & 0.55 \\
\hline & Pen & 8 & 2.41 & 0.30 & 1.06 & 0.21 & 0.32 \\
\hline & Residuals & 25 & 7.07 & 0.28 & & 0.62 & \\
\hline & Total & 40 & 11.38 & 0.86 & & 1.00 & \\
\hline
\end{tabular}

\section{Figure captions}

Figure 1. Map showing the stock source of the male Chinook salmon used for fertilization of eight pure and hybrid crosses used in this study. Crosses included pure (YIAL) and hybrid (CAP, CHILL, NIT, PUNT, RC, BQ, QUIN) crosses of Chinook salmon. Abbreviations:Robertson Creek "RC", Big Qualicum River "BQ", Capilano River "CAP", Chilliwack River "CHILL", Nitinat River "NIT", Puntledge River "PUNT", Quinsam River "QUIN".

Figure 2. Mean ( \pm 1 SEM) Shannon's index and Chao I across all breeding crosses. Significant cross differences $(\mathrm{P}<0.05)$ from the grand mean were not found for Chao I or Shannon's index. No statistically significant pairwise differences between stocks were found for either alpha diversity metric. Hybrid cross abbreviations are defined in Figure 1.

Figure 3. Principal coordinate analysis $(\mathrm{PCoA})$ plot with the first two PCoA values. PCoA used pairwise Bray-Curtis distances across all gut microbiome samples from Chinook salmon hybrid offspring with sires from each identified source population (see Fig. 1). Each open circle represents the average PCoA coordinates for a breeding cross, and error bars represent 95\% confidence intervals (CI). Hybrid cross abbreviations are defined in Figure 1.

Figure 4. Unique OTU analysis presenting the largest interaction sizes and the associated hybrid crosses of Chinook salmon used in this study. OTUs occurring less than 3 times (tripletons) in the dataset were removed prior to the analysis. Overall, 1,239 OTUs commonly occurred in all crosses. CHILL, YIAL, and BQ were the stocks with the greatest number of unique OTUs. Conversely, YIAL, QUIN, and RC showed the greatest number of missing OTUs commonly found in other crosses. Hybrid cross abbreviations are defined in Figure 1.

Figure 5. Histograms showing relative frequencies of candidate gut microbiome OTUs across all eight Chinook salmon hybrid crosses. Shown are 13 OTUs that showed significant differences among the crosses. Error bars represent standard error of the mean. Letters above the error bars represent post-hoc pairwise statistical differences among crosses, based on multiple student $\mathrm{T}$ tests of the mean $(\mathrm{P}<0.05)$, adjusted for multiple comparisons with BH. Hybrid cross abbreviations are defined in Figure 1. 
Figure 6. Histograms showing relative frequencies of candidate gut microbiome taxa families across all eight Chinook salmon hybrid crosses. Shown are seven taxonomic families that showed significant differences among the crosses. Error bars represent standard error of the mean. Letters above the error bars represent post-hoc pairwise statistical differences among crosses, based on multiple student $\mathrm{T}$ tests of the mean $(\mathrm{P}<$ 0.05), adjusted for multiple comparisons with BH. Hybrid cross abbreviations are defined in Figure 1.
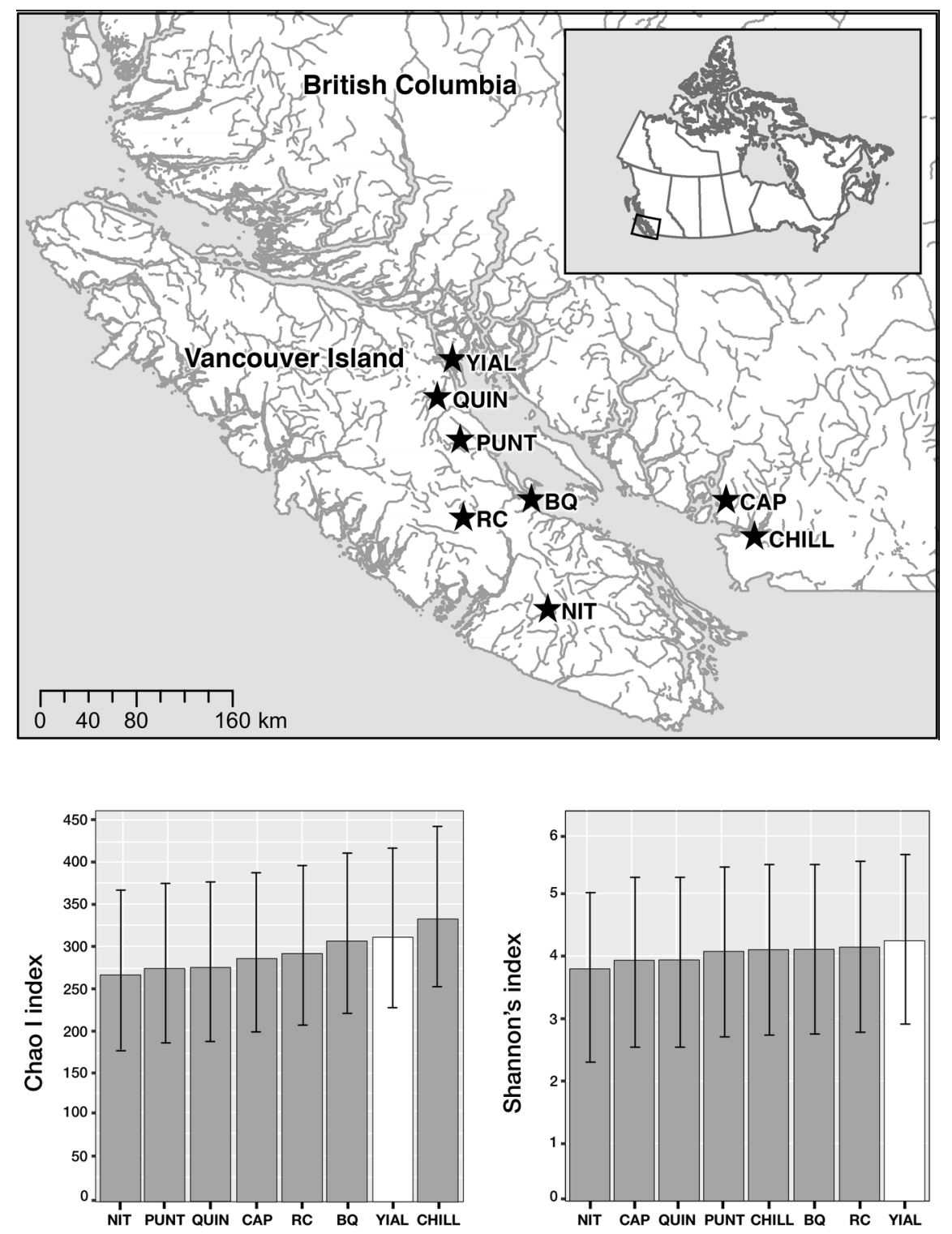

Hybrid cross 

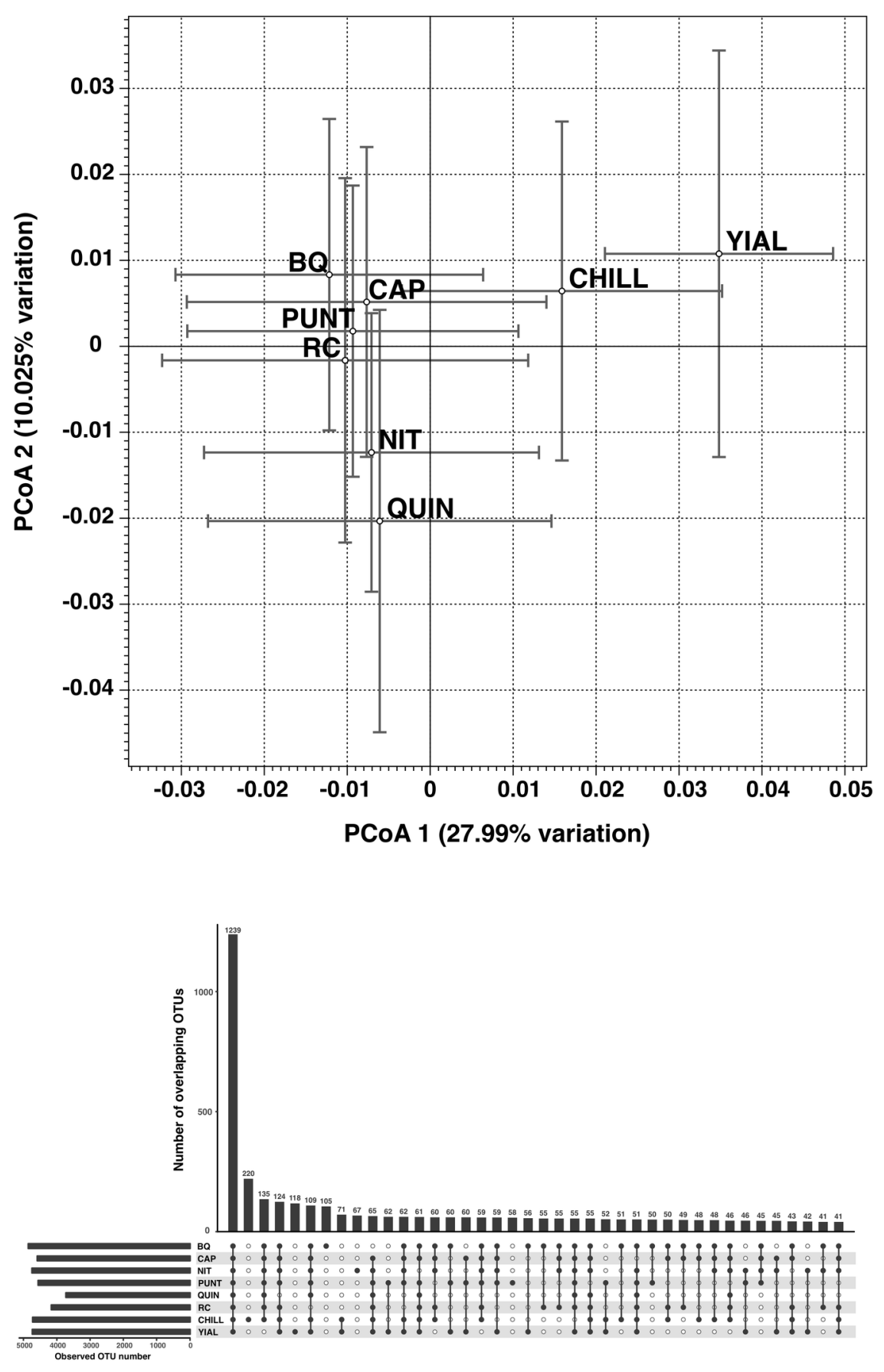


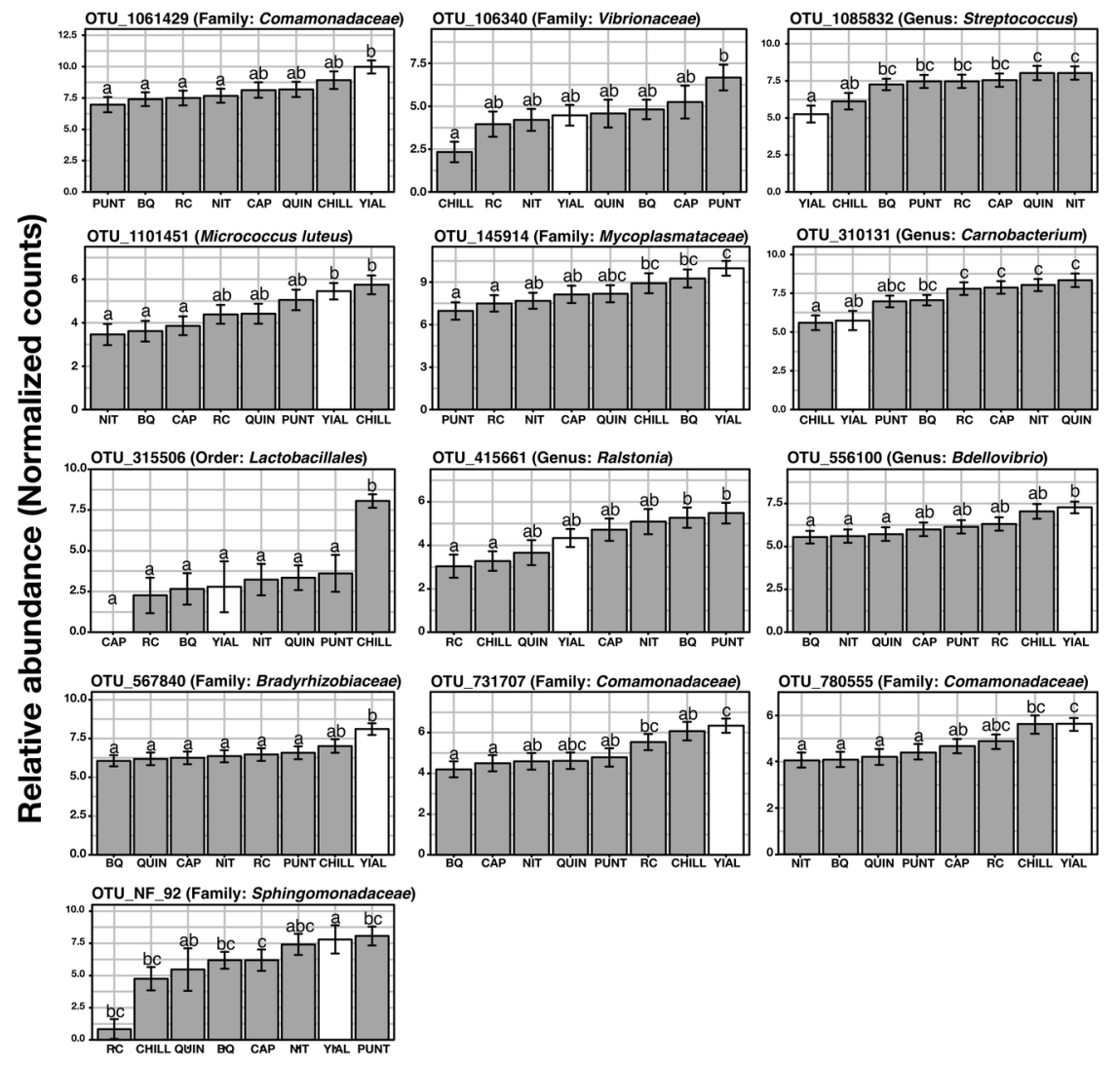

Population hybrid cross 


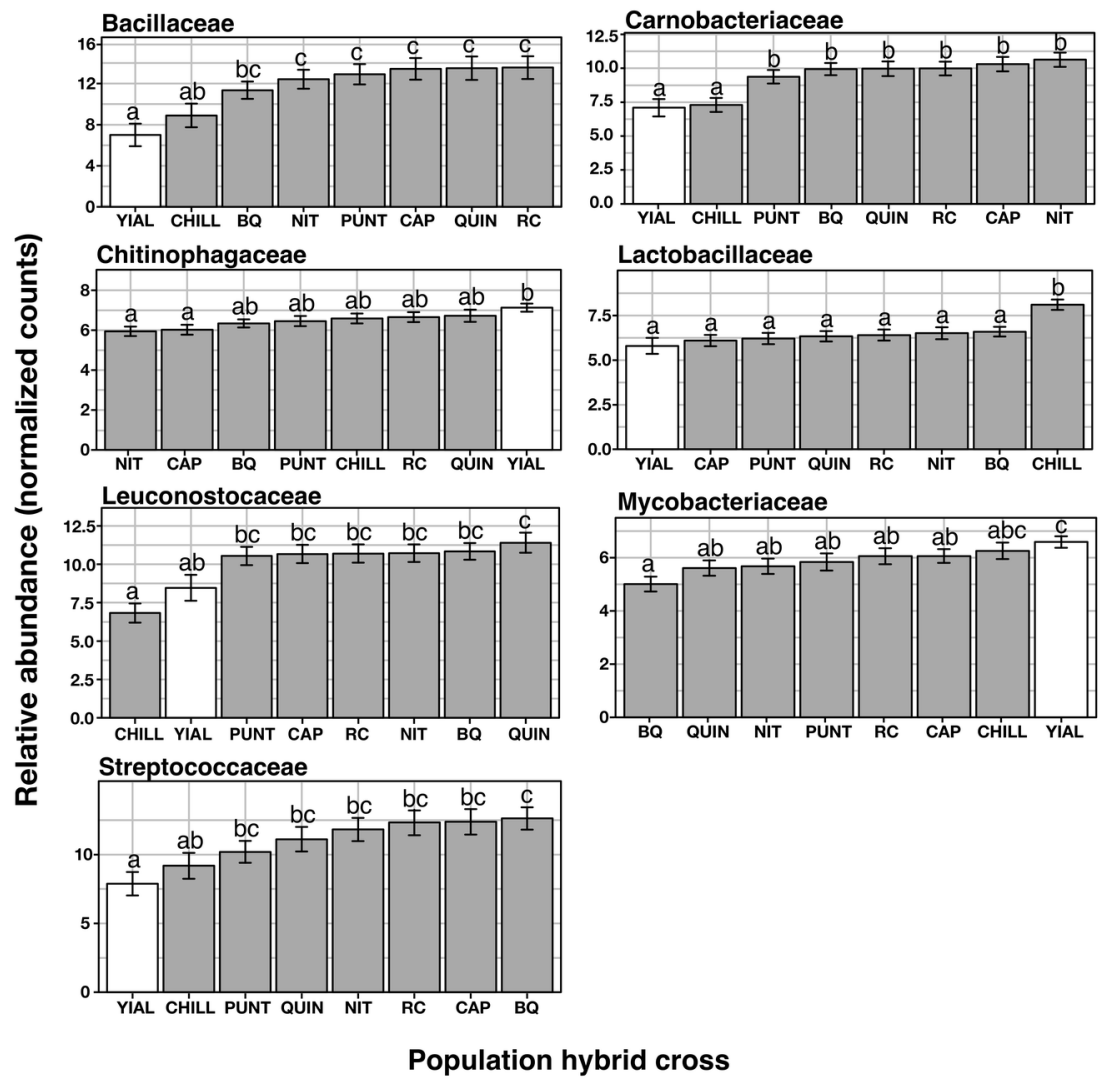

